\title{
THE
}

$11-27-2012$

\section{Rare-Event Sampling: Occupation-Based Performance Measures for Parallel Tempering and Infinite Swapping Monte Carlo Methods}

\author{
J. D. Doll \\ Nuria Plattner \\ David L. Freeman \\ University of Rhode Island, dfreeman@uri.edu \\ Yufei Lu \\ Paul Dupuis
}

Follow this and additional works at: https://digitalcommons.uri.edu/chm_facpubs

Terms of Use

All rights reserved under copyright.

\section{Citation/Publisher Attribution}

Doll, J. D., Plattner, N., Freeman, D. L., Liui, Y., \& Dupuis, P. (2012). Rare-Event Sampling: Occupation-Based Performance Measures for Parallel Tempering and Infinite Swapping Monte Carlo Methods. Journal of Chemical Physics, 137(20), \#204112. doi: 10.1063/1.4765060

Available at: http://dx.doi.org/10.1063/1.4765060

This Article is brought to you for free and open access by the Chemistry at DigitalCommons@URI. It has been accepted for inclusion in Chemistry Faculty Publications by an authorized administrator of DigitalCommons@URI. For more information, please contact digitalcommons-group@uri.edu. 


\section{AIP $\begin{gathered}\text { mesoumalot } \\ \text { chemical Physics }\end{gathered}$}

Rare-event sampling: Occupation-based performance measures for parallel tempering and infinite swapping Monte Carlo methods

J. D. Doll, Nuria Plattner, David L. Freeman, Yufei Liu, and Paul Dupuis

Citation: J. Chem. Phys. 137, 204112 (2012); doi: 10.1063/1.4765060

View online: http://dx.doi.org/10.1063/1.4765060

View Table of Contents: http://jcp.aip.org/resource/1/JCPSA6/v137/i20

Published by the American Institute of Physics.

Additional information on J. Chem. Phys.

Journal Homepage: http://jcp.aip.org/

Journal Information: http://jcp.aip.org/about/about_the_journal

Top downloads: http://jcp.aip.org/features/most_downloaded

Information for Authors: http://jcp.aip.org/authors

\section{ADVERTISEMENT}
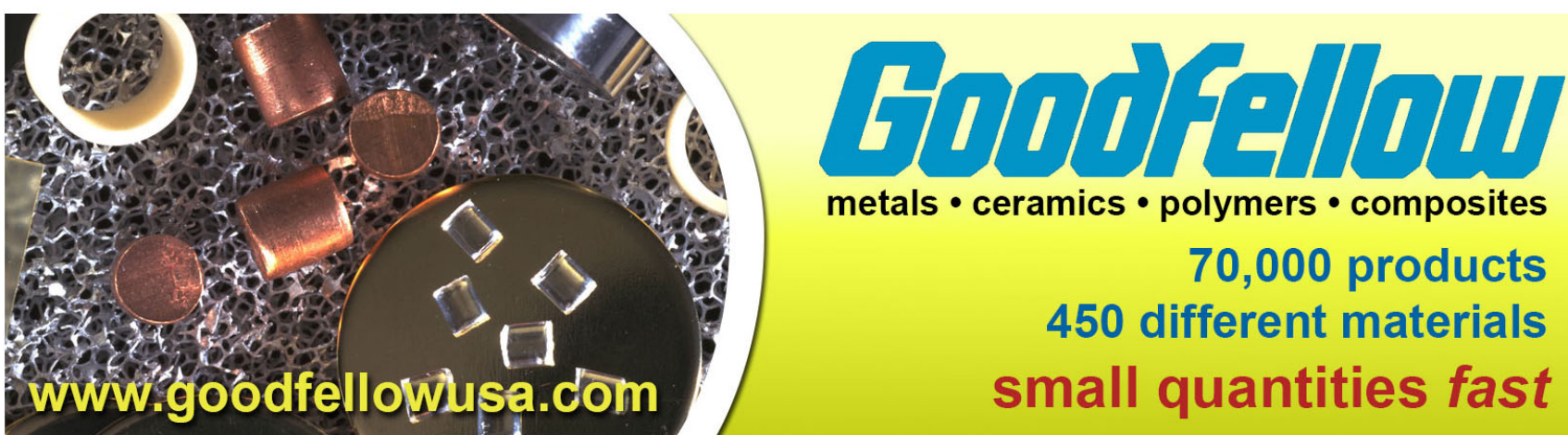
metals • ceramics $\bullet$ polymers $\bullet$ composites

70,000 products 450 different materials small quantities fast 


\title{
Rare-event sampling: Occupation-based performance measures for parallel tempering and infinite swapping Monte Carlo methods
}

\author{
J. D. Doll, ${ }^{1}$ Nuria Plattner, ${ }^{1,2}$ David L. Freeman, ${ }^{3}$ Yufei Liu, ${ }^{4}$ and Paul Dupuis ${ }^{4}$ \\ ${ }^{1}$ Department of Chemistry, Brown University, Providence, Rhode Island 02912, USA \\ ${ }^{2}$ Department of Chemistry, University of Basel, Klingelberegstrasse 80, CH-4056 Basel, Switzerland \\ ${ }^{3}$ Department of Chemistry, University of Rhode Island, Kingston, Rhode Island 02881, USA \\ ${ }^{4}$ Division of Applied Mathematics, Brown University, Providence, Rhode Island 02912, USA
}

(Received 31 August 2012; accepted 17 October 2012; published online 27 November 2012)

\begin{abstract}
In the present paper we identify a rigorous property of a number of tempering-based Monte Carlo sampling methods, including parallel tempering as well as partial and infinite swapping. Based on this property we develop a variety of performance measures for such rare-event sampling methods that are broadly applicable, informative, and straightforward to implement. We illustrate the use of these performance measures with a series of applications involving the equilibrium properties of simple Lennard-Jones clusters, applications for which the performance levels of partial and infinite swapping approaches are found to be higher than those of conventional parallel tempering. (C) 2012 American Institute of Physics. [http://dx.doi.org/10.1063/1.4765060]
\end{abstract}

\section{INTRODUCTION}

Monte Carlo methods ${ }^{1-4}$ constitute an important and versatile set of tools for the study of many-body systems. By providing a refinable means for extracting macroscopic properties from specified microscopic force laws, such methods permit the atomistic study of systems of realistic physical complexity without the need for the introduction of uncontrollable approximations.

Although robust and general purpose, important practical issues can arise in the application of Monte Carlo techniques. ${ }^{2-4}$ One such matter is the general problem of rareevent sampling. In typical equilibrium applications, where the relevant numerical task involves estimating averages of interest over known probability distributions, the adequacy of the sampling methods involved is an obvious and critical issue. If the probability distribution has a single, simply connected region of importance, ordinary random walk sampling procedures ${ }^{1,3-5}$ are generally adequate. If, however, the distribution in question contains multiple, isolated regions of importance, transitions between them can become infrequent (rare) when using conventional Metropolis-style methods rendering the associated property estimates unreliable. Unfortunately, from a practical point of view, applications in which such rare-event difficulties arise are themselves not rare. They arise frequently, for example, in studies of activated processes, ${ }^{6}$ applications of substantial importance in chemical, biological, and materials investigations.

A number of approaches have been devised in an effort to overcome rare-event sampling concerns. One of the more widely used is the parallel tempering ${ }^{7,8}$ (PT) or replica exchange technique. ${ }^{9}$ As summarized in Ref. 6, this method utilizes an expanded computational ensemble composed of systems corresponding to a range of control parameters such as the temperature. The core idea is to make use of information produced by one portion of the ensemble (e.g., higher temperatures) to improve the sampling in another (e.g., lower temperatures). The information transfer needed to improve the sampling is generally accomplished by augmenting conventional Metropolis-style particle displacements with suitably designed "swaps" of coordinates between ensemble temperatures. Strategies for the selection of the ensemble temperatures ${ }^{10-14}$ as well as for the frequency ${ }^{15,16}$ and nature ${ }^{17-20}$ of the swap attempts have been discussed.

Recently, a large deviation analysis of the performance of parallel tempering has led to the development of a new class of rare-event methods, the infinite swapping (INS) approach. ${ }^{21,22}$ In its most complete form the INS method can be viewed as the extreme limit of parallel tempering in which swaps involving all possible temperatures are attempted at an infinitely rapid rate, a limit the large deviation analysis demonstrates to be optimal. The infinitely rapid swaps induce a Born-Oppenheimer like environment, one in which the relevant distribution becomes a thermally symmetrized ana$\log$ of that used in conventional parallel tempering. Although the computational requirements for the full INS method grow rapidly with the number of temperatures involved, practical methods that capture a substantial level of the performance potential of the full approach while offering a significant cost/performance increase relative to parallel tempering are available. Details of these partial infinite swapping (PINS) approaches and their implementation are discussed in Refs. 21 and 22.

Performance measures are important tools in the development and application of rare-event sampling methods. Such measures are necessary, for example, for making decisions concerning questions ranging from whether or not a particular method is "working" to those related to the relative performance of alternative approaches or implementations. Ideally, the measures in question should be informative, straightforward to implement, and of sufficient generality that their utility transcends specific systems or properties. In practice, since approaches may differ with respect to these 
desirable features, having a variety of performance measures available is useful.

One general strategy for approaching the discussion of performance measures is to identify criteria that are plausibly related to the functioning of the computational method involved and to utilize those criteria to "optimize" the associated simulation. In the case of the parallel tempering approach, for example, tempering swaps are at the core of the method and are thus a reasonable focus for attention. Kofke has posited that achieving a uniform acceptance probability for such swap attempts across the computational ensemble is a desirable goal, one that provides a means for selecting ensemble temperatures. ${ }^{10,23}$ In their work Predescu and coworkers ${ }^{11,12}$ have expanded this line of argument by linking the acceptance probabilities involved to system heat capacity information. A somewhat different approach, explored by Katzgraber et al. ${ }^{14}$ is based on the premise that in a parallel tempering application it is the rate at which configurations transit the computational ensemble that is of primary interest. Applications have shown that the tempering ensembles produced by the uniform acceptance and maximum rate approaches are in general not identical.

Rather than beginning with the identification of an optimization criterion, another approach in the discussion of performance measures is to identify a property (or properties) inherent in the simulation method itself and to utilize the presence/absence/rate of achievement of this property as a performance measure. Neirotti et al. ${ }^{24}$ adopt such an "inherent property" approach in their study of rare-event sampling. Building upon earlier work by Thirumalai and co-workers, ${ }^{25}$ they utilize the decay of energy-related metrics to known limiting values to monitor sampling.

In the present paper we explore the development and application of occupation-based approaches for temperingbased Monte Carlo methods. The outline of the paper is as follows. We begin in Sec. II with an empirical observation that points to a somewhat surprising property of parallel tempering methods. After demonstrating the rigorous and general nature of this result, we use the property involved to develop a number of convenient performance diagnostics for both parallel tempering and PINS methods. In Sec. III we illustrate the use of the resulting diagnostics for a number of numerical examples of varying complexity that involve the equilibrium properties of models of simple rare-gas clusters. Section IV contains a summary and discussion of our results.

\section{BACKGROUND AND AN OBSERVATION}

We begin by considering a number of tempering investigations of a particular system, a Lennard-Jones model of a 13 atom rare-gas cluster. In addition to providing a concrete framework for the present discussion, these simple cluster simulations contain hints of features that prove useful in the broader consideration of rare-event sampling. We find it convenient to express the results with reference to a particular system (argon) thereby giving a physical context to the observed features noting that computed properties for the Lennard-Jones model are universal and can be expressed using reduced variables.
Occupation traces of the type introduced by Katzgraber et al. ${ }^{14}$ are a convenient device for discussing tempering based sampling methods. Such traces are a record of the temperature associations for a particular coordinate set as it moves within the tempering ensemble during the simulation. For parallel tempering applications there are clear temperature-coordinate associations at each step in the simulation. For infinite and PINS swapping approaches, on the other hand, such explicit associations are obscured by the symmetrization involved. Although this makes the identification and construction of occupation traces for INS and PINS applications a somewhat more subtle issue, a generalization of the basic parallel tempering result proves both possible and practical. The key is to base such a generalization on steps in the sampling process for which the temperature-coordinate associations are unambiguous.

Sampling in the present work is based on the "dualchain" approach, a method that is described in detail in Refs. 21 and 22. This technique involves partitioning the ensemble temperatures into contiguous blocks in two distinct ways (dual chains). Although symmetrization in this approach is "partial" (i.e., occurs only within the various separate temperature blocks), by suitably combining moves within the individual chains with others that exchange information between them it is possible to produce a sampling of the fully symmetrized, infinite swapping distribution. Most importantly, this sampling is accomplished without the factorialscale growth in computational effort that would be incurred in a brute-force INS approach. The "hand-off" or transfer of information between the two sampling chains is a critical step in the dual-chain process. During such hand-offs explicit temperature-coordinate associations are established, associations that provide a natural basis for the construction of INS and PINS occupation traces and that are utilized for such tasks in the present work.

Figure 1 shows a number of occupation traces obtained from short, three-temperature simulations of the $\mathrm{Ar}_{13}$ cluster. The traces displayed chronicle the progress of configurations that are initially at the lowest of the three ensemble
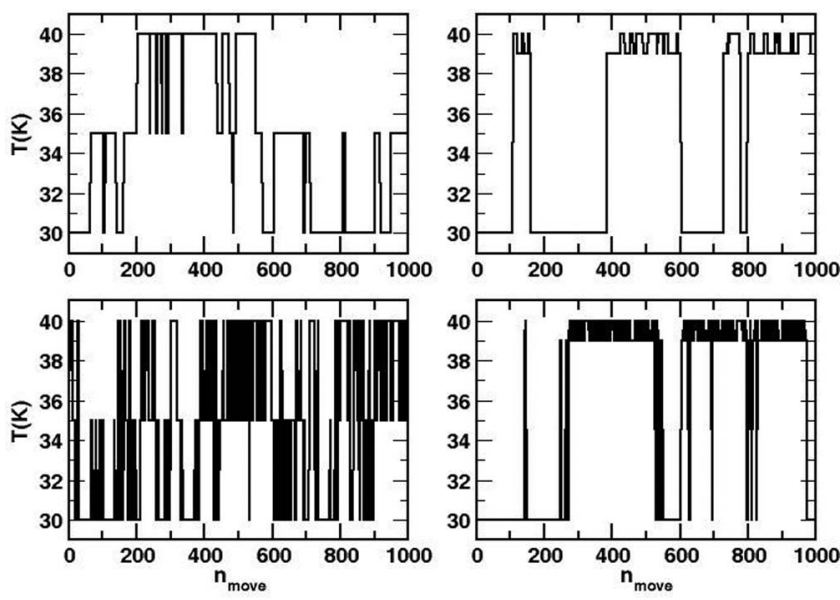

FIG. 1. Occupation traces for three-temperature PT (top row) and PINS (bottom row) simulations for $\mathrm{Ar}_{13} \cdot \mathrm{T}_{1}=30 \mathrm{~K}$ and $\mathrm{T}_{3}=40 \mathrm{~K}$ for all simulations, while $\mathrm{T}_{2}=35 \mathrm{~K}$ for simulations in left column and $39 \mathrm{~K}$ for those in right column. 
temperatures for a period of 1000 moves. Analogous traces for configurations initially associated with either of the other two ensemble temperatures could be similarly constructed. The low and high-temperatures used in all simulations in Fig. 1 are $\mathrm{T}_{1}=30 \mathrm{~K}, \mathrm{~T}_{3}=40 \mathrm{~K}$, respectively, values chosen to bracket the temperature of the $\operatorname{Ar}_{13}$ cluster's heat capacity maximum $(34 \mathrm{~K}) .{ }^{26}$ The intermediate temperature, $\mathrm{T}_{2}$, varies being $35 \mathrm{~K}$ for the simulations in the left column of Fig. 1 and $39 \mathrm{~K}$ for those in the right column. Results in the top row of Fig. 1 correspond to PT simulations, while those in the bottom row are analogous, three-temperature PINS results. Unless otherwise stated all numerical simulations in the present work utilize the methods described in Appendix A and in Refs. 21 and 22. The dual chains in the PINS simulations have one chain with temperature blocks that consist of $\mathrm{T}_{1}$ and $\mathrm{T}_{2-3}$ and another chain with blocks that consist of $\mathrm{T}_{1-2}$ and $\mathrm{T}_{3}$. We see in Fig. 1 that the movement of the system in question throughout the computational ensemble is sensitive to both ensemble choice ( $T_{2}$ value) and to sampling method (PT or PINS).

Trebst et al. ${ }^{14,27}$ have suggested that the number of sampling moves required to traverse the computational ensemble provides a convenient measure of sampling performance. This idea is illustrated for the present application in Fig. 2 where the average number of moves required for the system to make a round trip across the various computational ensembles, $\left\langle\mathrm{n}_{\mathrm{rt}}\right\rangle$, is plotted as a function of the choice of the intermediate ensemble temperature, $\mathrm{T}_{2}$, for the various methods. These results are obtained from occupation traces that contain a total of $2^{17}$ moves. As anticipated, there is a minimum in $\left\langle\mathrm{n}_{\mathrm{rt}}\right\rangle$ values as a function of the choice of $T_{2}$ for the various sampling methods. The "optimal" $\mathrm{T}_{2}$ choice for this system (i.e., the value that produces the most rapid traversal of the computational ensemble) is $\sim 35 \mathrm{~K}$ for both PT and PINS methods. For a specified value of $\mathrm{T}_{2}$, on the other hand, we see in Fig. 2 that the movement across the computational ensemble is appreciably more rapid with the INS and PINS methods than with parallel tempering. For example, for $\mathrm{T}_{2}=35 \mathrm{~K}$ the PT, PINS, and INS $\left\langle\mathrm{n}_{\mathrm{rt}}\right\rangle$ values are approximately 298.6, 61.2, and

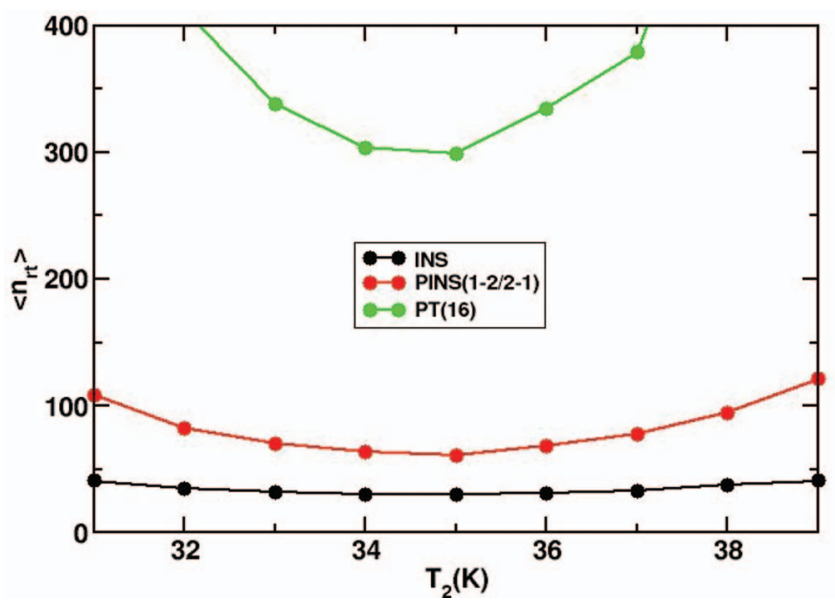

FIG. 2. Plots of the average number of moves required for a round-trip transit of the computational ensemble, $\left\langle\mathrm{n}_{\mathrm{rt}}\right\rangle$ as function of $\mathrm{T}_{2}$, for extended versions of the three-temperature $\mathrm{Ar}_{13}$ simulations of the type in Fig. 1.
TABLE I. Observed fractions of total moves $\left(f_{n}\right)$ spent at each of the ensemble temperatures by two three-temperature $\mathrm{Ar}_{13}$ parallel tempering (PT) and partial swapping (PINS) simulations. In both ensembles, $\mathrm{T}_{1}=30 \mathrm{~K}$ and $\mathrm{T}_{3}=40 \mathrm{~K}$, while in one ensemble $\mathrm{T}_{2}=35 \mathrm{~K}$ and in the other $\mathrm{T}_{2}=39 \mathrm{~K}$.

\begin{tabular}{cccccc}
\hline \hline $\mathrm{T}_{\mathrm{n}}$ & $\mathrm{f}_{\mathrm{n}}(\mathrm{PT})$ & $\mathrm{f}_{\mathrm{n}}(\mathrm{PINS})$ & $\mathrm{T}_{\mathrm{n}}$ & $\mathrm{f}_{\mathrm{n}}(\mathrm{PT})$ & $\mathrm{f}_{\mathrm{n}}(\mathrm{PINS})$ \\
\hline 30 & 0.3314 & 0.3317 & 30 & 0.3342 & 0.3348 \\
35 & 0.3330 & 0.3329 & 39 & 0.3334 & 0.3324 \\
40 & 0.3356 & 0.3354 & 40 & 0.3324 & 0.3328 \\
\hline \hline
\end{tabular}

29.9, respectively. As an aside, the "flatter" nature of the INS and PINS plots in Fig. 2 suggests that the performance of such methods is more robust than that of parallel tempering with respect to sub-optimal choices of the computational ensemble.

We now turn to a somewhat unexpected aspect of the tempering results in Fig. 1. Beyond its intrinsic interest, this feature provides the basis for a number of simple, propertyindependent tools that prove useful in the characterization and analysis of rare-event sampling issues. Table I examines the fraction of moves various three-temperature $\mathrm{Ar}_{13}$ occupation traces of the type in Fig. 1 spend in the different temperature streams. The occupation traces used to generate these results are longer ( $2^{23}$ total moves), but otherwise of the type shown in Fig. 1. The numerical results of Table I suggest that the fractional occupancies of the various temperature streams are in fact uniform for all the tempering methods involved regardless of the choice of $\mathrm{T}_{2}$. Such uniformity turns out to be a general and rigorous property of INS, PINS, and parallel tempering approaches and holds for an arbitrary number of temperatures. That is, if there are $\mathrm{N}_{\mathrm{t}}$ temperatures in the computational ensemble, the fraction of the total number of moves such occupation traces spend in any particular ensemble temperature asymptotically approaches a value of $1 / \mathrm{N}_{\mathrm{t}}$. This "equal occupancy" result is one of the principal findings of the current work.

Although the property appears not to have been noticed previously, it turns out that the equal occupancy result suggested by Table I is a general feature of the parallel tempering method. We provide a heuristic argument for this claim below. A more detailed argument as well as extensions to cover both infinite and PINS approaches are given in Appendix B.

We begin by considering an $\mathrm{M}$-step parallel tempering simulation that involves $\mathrm{N}_{\mathrm{t}}$ temperatures, $\left\{\mathrm{T}_{\mathrm{n}}\right\}, \mathrm{n}=1, \mathrm{~N}_{\mathrm{t}}$. As they move about in the computational ensemble, the initial configurations in such a simulation generate $\mathrm{N}_{\mathrm{t}}$ distinct occupation traces, one for each of the possible initial temperatures. If we designate the temperatures for one such trace, labeled $\alpha$, at step $\mathrm{m}$ in the simulation as $\mathrm{T}_{\alpha}(\mathrm{m})$, then the fraction of moves trace- $\alpha$ spends in temperature stream $\mathrm{T}_{\mathrm{n}}, \mathrm{f}_{\mathrm{n}}^{\alpha}$, is given by

$$
\mathrm{f}_{\mathrm{n}}^{\alpha}=\frac{1}{\mathrm{M}} \sum_{\mathrm{m}=1}^{\mathrm{M}} 1_{\mathrm{T}_{\alpha}(\mathrm{m}), \mathrm{T}_{\mathrm{n}}}
$$

where

$$
\mathbf{1}_{\mathrm{T}_{\alpha}(\mathrm{m}), \mathrm{T}_{\mathrm{n}}}=\left\{\begin{array}{ll}
1, & \mathrm{~T}_{\alpha}(\mathrm{m})=\mathrm{T}_{\mathrm{n}} \\
0, & \text { otherwise }
\end{array} .\right.
$$


The underlying occupation traces are stationary, ergodic random processes. Consequently, the asymptotic statistical properties of the $\mathrm{N}_{t}$ possible occupation traces arising from the various starting locations must be equivalent (i.e., there can be no dependence of the $\mathrm{f}_{\mathrm{n}}^{\alpha}$ values on the index $\alpha$ ). As a result the values of $\mathrm{f}_{n}^{\alpha}$ for a given temperature approach a common value, $\mathrm{f}_{\mathrm{n}}$, for all $\mathrm{N}_{\mathrm{t}}$ possible occupation traces. Since we know that the $f_{n}^{\alpha}$ values are all asymptotically equal for all values of $\alpha$, and since we know from Eq. (2.1) that their sum over $\alpha$ for a given temperature totals unity, we conclude that $f_{n}=1 / N_{t}$ for any temperature $T_{n}$. Other than assuming it to be non-zero, the equal occupancy result does not depend on a particular choice for the parallel tempering swap attempt frequency. As noted in the Introduction, the infinite swapping approach can be viewed as a limiting form of parallel tempering in which swaps involving all possible temperatures are attempted at an infinitely rapid rate. One thus suspects (properly) that the parallel tempering equal occupancy result is also valid for INS and PINS approaches. A demonstration of this general result is presented in Appendix B.

The uniform occupation property of the parallel tempering, PINS, and INS methods provides the basis of a number of useful sampling performance measures. One such measure is the Shannon entropy associated with the occupation fractions for the simulation. If the occupation fraction for temperature stream $T_{n}$ after $n_{\text {move }}$ simulation moves is designated by $f_{n}\left(n_{\text {move }}\right)$, then the occupation entropy for the simulation, $S_{f}$, is defined as

$$
\mathrm{S}_{\mathrm{f}}=-\sum_{\mathrm{n}=1}^{\mathrm{N}_{\mathrm{t}}} \mathrm{f}_{\mathrm{n}}\left(\mathrm{n}_{\text {move }}\right) \ln \left[\mathrm{f}_{\mathrm{n}}\left(\mathrm{n}_{\text {move }}\right)\right] .
$$

When $\mathrm{n}_{\text {move }}=1, \mathrm{f}_{\mathrm{n}}\left(\mathrm{n}_{\text {move }}\right)=\delta_{\mathrm{n}, \mathrm{s}}$, where $\mathrm{s}$ is the index of the initial temperature stream of the trace. So defined, the $S_{\mathrm{f}}$ value begins at zero and increases as the simulation proceeds. Because the asymptotic limit of $S_{f}$ is known (i.e., $S_{\max }=\ln \left(\mathrm{N}_{\mathrm{t}}\right)$ ), both the convergence and the rate of convergence to this limit can serve as practical performance diagnostics. At the crudest level the failure of $S_{\mathrm{f}}$ to achieve its known limiting value signals an obvious breakdown in the sampling. More generally, the rate of approach of $S_{f}$ to this limit provides a convenient, quantitative, property-independent means for comparing the performance of different computational ensembles and/or sampling methods.

Figures 3 and 4 contain plots of the entropies computed from the PT and PINS occupation traces for $\mathrm{Ar}_{13}$ from Table I. We see in Fig. 3 that the entropies for all simulations considered appear to achieve their proper limiting values, which in this case is $\ln (3)$. We also see in Fig. 4 that the rate at which this limit is achieved for a specified intermediate temperature is more rapid with the PINS method than with parallel tempering. In fact, judged by this entropic measure the results of Fig. 4 indicate that the PINS performance for a "bad" choice of intermediate temperatures $\left(\mathrm{T}_{2}=39 \mathrm{~K}\right)$ is actually superior to that of parallel tempering for the optimal choice $\left(\mathrm{T}_{2}=35 \mathrm{~K}\right)$.

A related performance measure is the fluctuation autocorrelation function of the occupation trace index. If we define the index of the temperature at step $m$ in an occupation trace

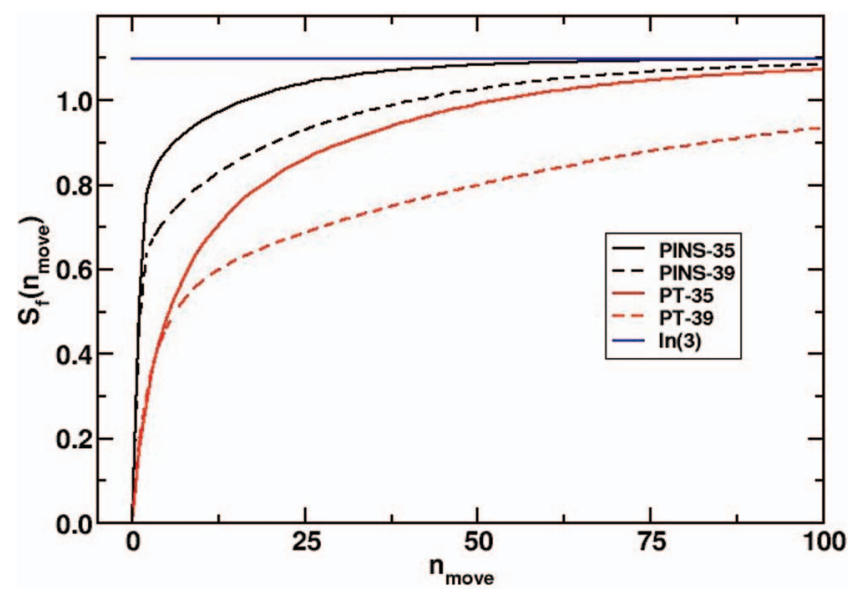

FIG. 3. Approach of $\mathrm{S}_{\mathrm{f}}\left(\mathrm{n}_{\text {move }}\right)$ (c.f., Eq. (2.3)) to its uniform limiting value for the three-temperature PINS and PT simulations of $\mathrm{Ar}_{13}$ used in Table I and described in the text. $\mathrm{T}_{1}=30 \mathrm{~K}, \mathrm{~T}_{3}=40 \mathrm{~K}, \mathrm{~T}_{2}=35 \mathrm{~K}$ or $39 \mathrm{~K}$.

as $\mathrm{N}(\mathrm{m})$, where $1 \leq \mathrm{N} \leq \mathrm{N}_{\mathrm{t}}$, and the fluctuation of this value about its overall average as $\delta \mathrm{N}(\mathrm{m})$, then the correlation function, $\mathrm{C}(\mathrm{s})$, for the stationary random process $\delta \mathrm{N}(\mathrm{m})$ is defined as

$$
\mathrm{C}(\mathrm{s})=\frac{\langle\delta \mathrm{N}(\mathrm{m}) \delta \mathrm{N}(\mathrm{m}+\mathrm{s})\rangle}{\langle\delta \mathrm{N}(\mathrm{m}) \delta \mathrm{N}(\mathrm{m})\rangle},
$$

where the brackets in Eq. (2.4) denote an average over the occupation trace in question. Plots of $\mathrm{C}(\mathrm{s})$ for the various occupation traces of Table I are shown in Fig. 5. We see that for this rather simple application the performance rankings suggested by Fig. 5 are consistent with those in Figs. 3 and 4 as well as with those based on the round-trip transit measures shown in Fig. 2.

Although modest in the examples considered thus far, the utility of the occupation-based performance measures set forth in Eqs. (2.3) and (2.4) can become more significant when dealing with more challenging applications. An example is illustrated in Figs. 6 and 7. Figure 6 shows occupation traces for a 66-temperature $\mathrm{Ar}_{38}$ PINS and PT simulations, both of which begin in the highest temperature. The details of these and related simulations will be described in Sec. III. For

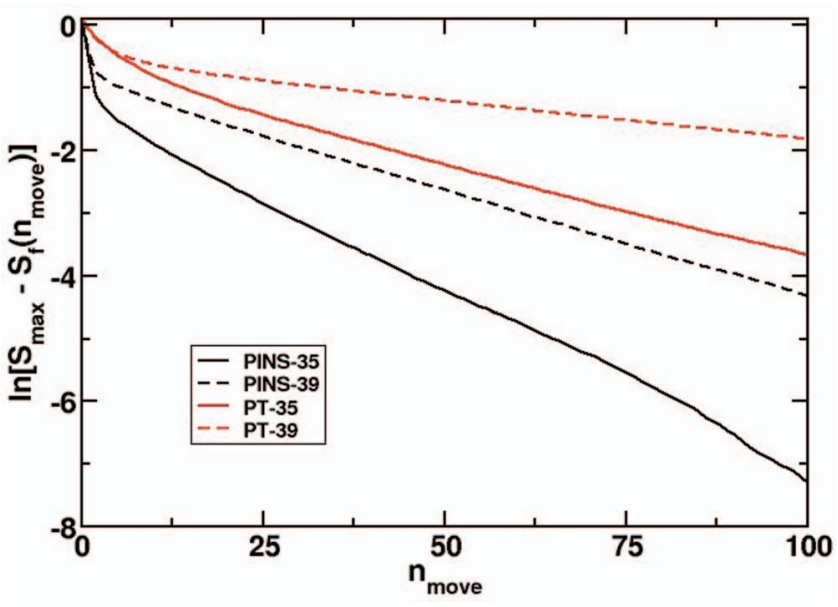

FIG. 4. Plot of $\ln \left(S_{\max }-S_{f}\right)$ for the three-temperature $\operatorname{Ar}_{13}$ results of Fig. 3. 


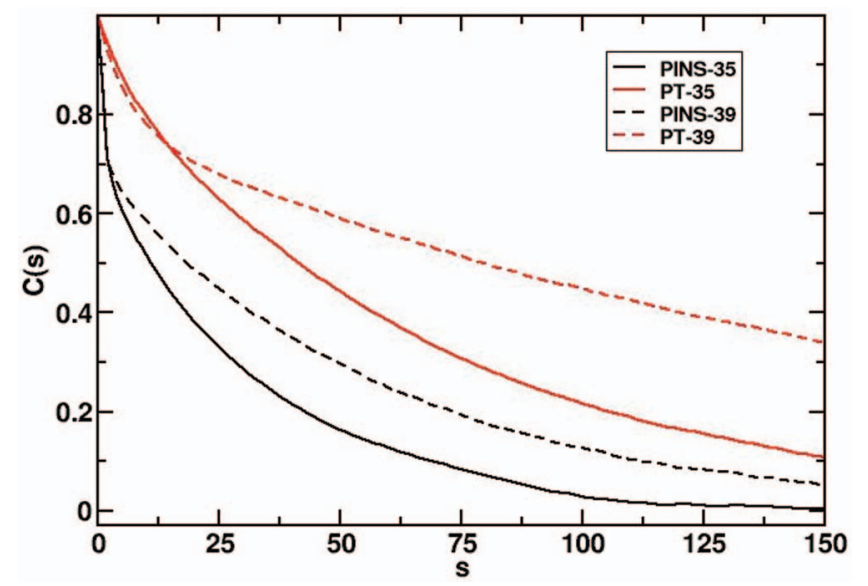

FIG. 5. Plots of C(s) (c.f., Eq. (2.4)) for the three-temperature $\mathrm{Ar}_{13}$ simulations of Fig. 3.

the moment the relevant feature to note in Fig. 6 is that over the course of the simulation the PINS occupation trace moves throughout the entire computational ensemble making a total of nine round trips across the $10-30 \mathrm{~K}$ interval, a range that includes both solid-like and liquid-like cluster behavior, while PT trace's movement is appreciably more limited. The failure of the PT occupation trace to complete even a single transit of the computational ensemble during the simulation interval shown is sufficient to raise questions concerning the adequacy of its associated sampling. In contrast, the ease and frequency with which the PINS occupation trace in Fig. 6 transits the ensemble could, if taken by itself, be viewed as evidence that all was in order and that the properties derived from the underlying simulation are reliable. The occupation analysis in Fig. 7, however, indicates that such a conclusion is premature. In particular, we see in Fig. 7 a significant underrepresentation of visits to the low-temperature portions of the ensemble. The occupation entropy for the distribution shown in Fig. 7 is $\mathrm{S}_{\mathrm{f}}=3.98$, well short of the $\ln (66)=4.19$ uniform limit. As discussed more fully in Sec. III, the underrepresentation of low-temperature visits seen in Fig. 7 is ultimately eliminated

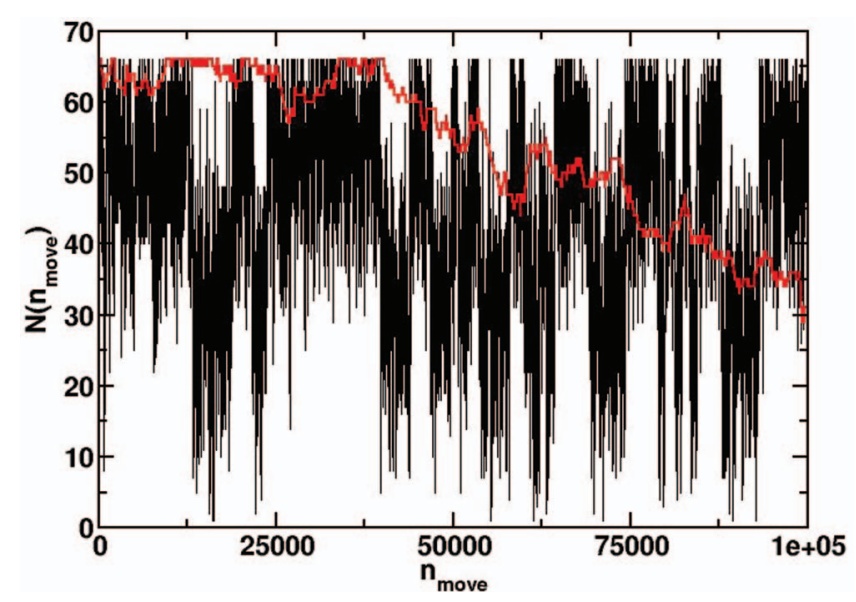

FIG. 6. A portion of occupation traces for 66-temperature $\operatorname{Ar}_{38}$ PINS (black) and PT (red) simulations discussed in the text. The vertical axis denotes the temperature index (1-66) as a function of the number of moves in the simulation.

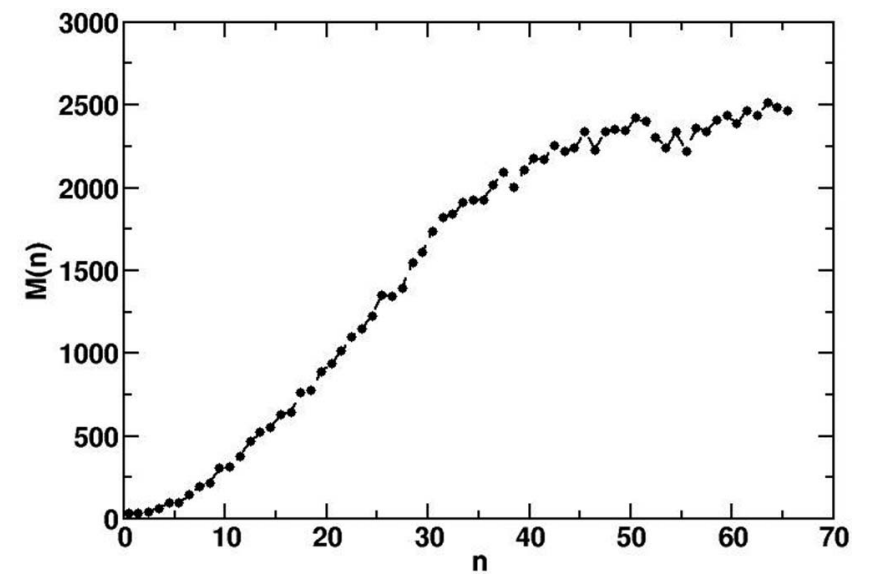

FIG. 7. A histogram of the PINS occupation trace shown in Fig. 6 showing the number of times the various temperature indices are visited, $M(n)$, as a function of $n$.

as more sampling points are included. The point we wish to emphasize, however, is that this underrepresentation is an indication of an important deficiency in the original, short simulation, a deficiency detected by the equal occupancy analysis, but not by transit-based measures.

In what follows it is useful to recast the difference between the actual and maximum occupation entropies in slightly different terms. Specifically, if we view the calculated $\mathrm{S}_{\mathrm{f}}$ value as arising from a uniform limit of a fictitious ensemble, the "active number" of temperatures in that ensemble, $\mathrm{N}_{\mathrm{a}}$, would be the exponential of the associated occupation entropy. In the case of Fig. $7 \mathrm{~N}_{\mathrm{a}}$ would thus be $\exp$ (3.98) or 53.5 , appreciably smaller than the actual number of 66 . We can also define a related quantity, the "active fraction" for the simulation, $\mathrm{F}_{\mathrm{a}}$, as the ratio of $\mathrm{N}_{\mathrm{a}}$ to the actual number of temperatures, $\mathrm{N}_{\mathrm{t}}$. In the example associated with Figs. 6 and $7, \mathrm{~F}_{\mathrm{a}}$ is $53.5 / 66$, or $\sim 0.810$. The concepts of active ensemble size and fraction will prove convenient in later discussions.

\section{ILLUSTRATIVE APPLICATIONS}

To explore further the utility of the performance measures developed in Sec. II, we consider their application to systems in which the number of temperatures in the computational ensemble and the physical complexity of the systems involved are increased.

Our first example is designed to illustrate how one can utilize the performance measures discussed in Sec. II to aid in the selection of both the tempering ensemble and the sampling method. Figures 8-11 contain various occupation entropy and autocorrelation function results for the $\mathrm{Ar}_{13}$ cluster. These are obtained with three different computational ensembles using both the PINS and PT methods outlined in Sec. II and Appendix A. The computational ensembles involved are each composed of a common number of temperatures, $\mathrm{T}_{1}-\mathrm{T}_{24}$, with $\mathrm{T}_{1}<\mathrm{T}_{2}, \ldots,<\mathrm{T}_{24}$ and $\mathrm{T}_{1}=20 \mathrm{~K}, \mathrm{~T}_{24}$ $=50 \mathrm{~K}$. Although they span the same overall range, the ensembles are chosen to have different temperature distributions. Specifically, in ensemble-a temperatures $\mathrm{T}_{1}-\mathrm{T}_{12}$ and $\mathrm{T}_{13}-\mathrm{T}_{24}$ uniformly cover the intervals $20-28 \mathrm{~K}$ and $42-50 \mathrm{~K}$, 


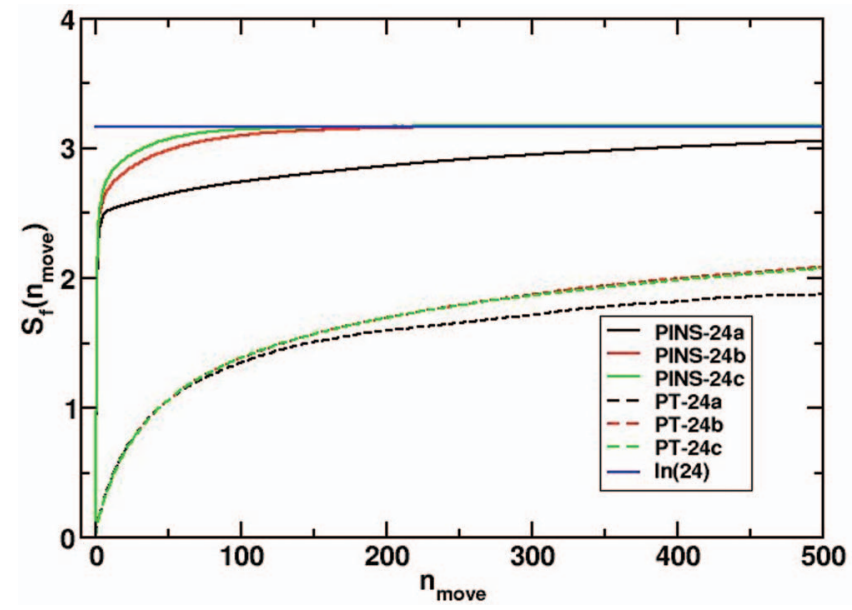

FIG. 8. A plot of $\mathrm{S}_{\mathrm{f}}\left(\mathrm{n}_{\text {move }}\right)$ obtained for $\mathrm{Ar}_{13}$ using PINS and PT methods for the various 24-temperature ensembles described in the text. The apparent "break" in the PINS-24a results occurs at an $\mathrm{S}_{\mathrm{f}}$ value of roughly 2.5 , a value that corresponds to an active number of temperatures $\left(\mathrm{N}_{\mathrm{a}}\right)$ of $\sim 12$.

respectively, while in ensemble-b the two, uniform 12temperature regions range from $20-32 \mathrm{~K}$ and $38-50 \mathrm{~K}$. The third ensemble, ensemble-c, consists of 24 temperatures uniformly distributed over the entire $20-50 \mathrm{~K}$ interval. The PINS results are obtained using dual-chain sampling methods of the type discussed previously. In these simulations one chain is composed of four symmetrized blocks of six temperatures $\left(\mathrm{T}_{1-6}, \mathrm{~T}_{7-12}, \mathrm{~T}_{13-18}, \mathrm{~T}_{19-24}\right)$, while the other chain consists of five symmetrized blocks $\left(\mathrm{T}_{1-3}, \mathrm{~T}_{4-9}, \mathrm{~T}_{10-15}, \mathrm{~T}_{16-21}, \mathrm{~T}_{22-24}\right)$. The PINS simulations utilize $2^{16}$ total moves, while the PT results utilize $2^{18}$. The other computational details for the PINS and PT simulations are discussed in Sec. II and Appendix A.

We see in Figs. 8 and 9 that for a given ensemble the rate of convergence of the PINS occupation entropies to the uniform, asymptotic limit of $\ln (24)$ is appreciably greater than that achieved with parallel tempering. We also see that the asymptotic convergence rate of the PINS results is highest for ensemble-c of the three considered. Interestingly, the initial $\left(\mathrm{small} \mathrm{n}_{\text {move }}\right.$ ) PINS convergence rates seen in Figs. 8 and 9 are similar for all three ensembles. As seen most clearly in Fig. 8,

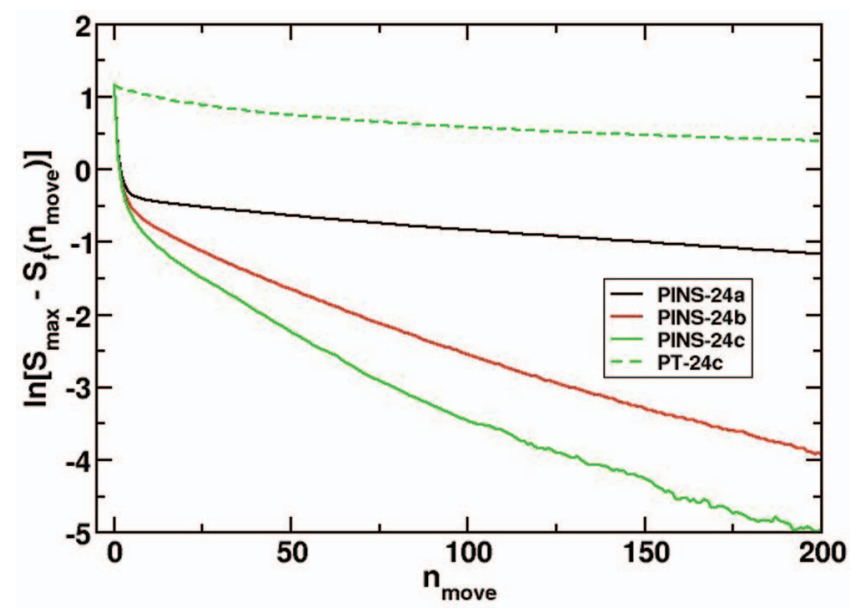

FIG. 9. Plot of $\ln \left(S_{\max }-S_{f}\right)$ for results in Fig. 8 .

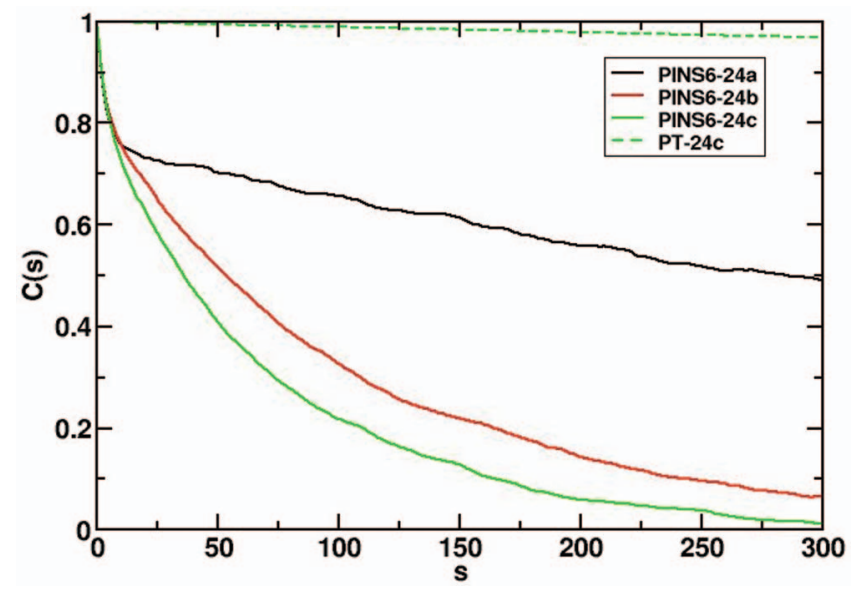

FIG. 10. C(s) for the PINS $\operatorname{Ar}_{13}$ results obtained using the three, 24temperature ensembles described in the text. PT results for ensemble-c are shown for comparison (dashed line near top of plot).

the PINS occupation entropy increase for ensemble-a is rapid up to a value of $\sim \mathrm{S}_{\mathrm{f}}=2.5$ after which a distinctly slower rate of increase is observed. This suggests $(\exp (2.5) \approx 12)$ that equilibration is rapid within the 12-temperature blocks of ensemble-a, but is ultimately hindered by the large temperature "gap" between $28-42 \mathrm{~K}$ in this ensemble. As with the analogous PINS results, the rate of approach of the occupation entropy to its uniform limit for the PT results in Fig. 8 is slowest for ensemble-a. While not identical, PT results for ensemble-b and ensemble-c are numerically quite similar for this system.

Figure 10 contains plots of the fluctuation autocorrelation functions, $\mathrm{C}(\mathrm{s})$, obtained from the various 24-temperature $\mathrm{Ar}_{13}$ simulations shown in Figs. 8 and 9. The plots in Fig. 10 reinforce the basic convergence story conveyed by the entropic measures and serve to illustrate the dramatic difference in the decay rates seen in the PINS and parallel tempering simulations. This difference is also conveyed by Fig. 11 where we plot a small portion of the PINS and PT occupation traces obtained for the best of the three 24-temperature
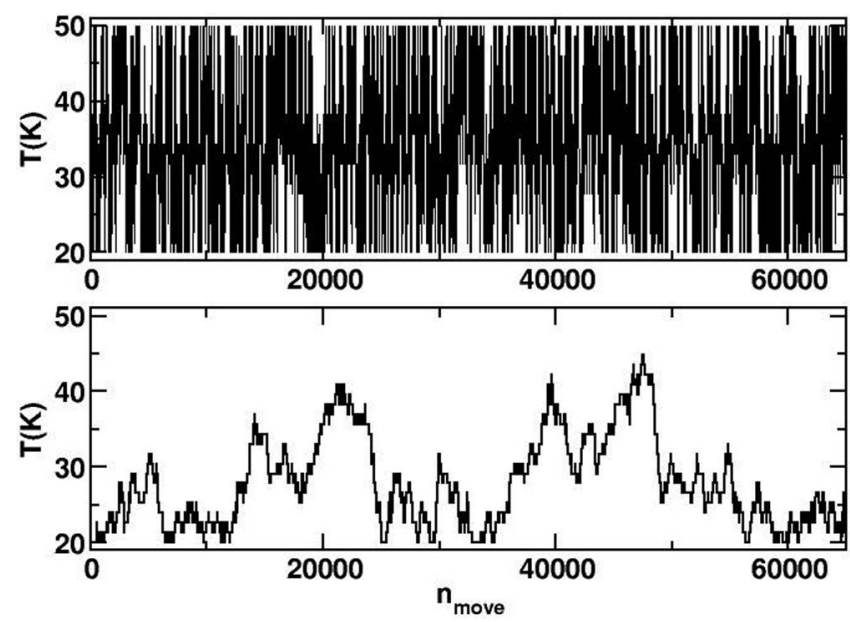

FIG. 11. Brief portions of occupation traces for PINS (top panel) and PT (bottom panel) simulations for $\mathrm{Ar}_{13}$ obtained using 24-temperature ensemble-c (see text for details). 
computational ensembles, ensemble-c. Although both PINS and PT traces show frequent passage through the temperature region of the heat capacity maximum $(34 \mathrm{~K})$, the qualitatively different character of the two traces provides a visual sense of the far greater rate of information transfer within the computational ensemble achieved by the PINS approach relative to the PT method for this application. This dramatic difference in the movement of the PINS and PT traces through the computational ensemble lies behind the vastly different rates of decay of the corresponding PINS and PT fluctuation autocorrelation functions seen in Fig. 10.

We now turn to our second, more challenging example, the 38-atom Lennard-Jones cluster. The LJ-38 system exhibits an interesting and diverse phenomenology, and, as a consequence, has received a significant amount of attention. ${ }^{28}$ As discussed in the pioneering work of Doye et al. ${ }^{29}$ this cluster has a double-funnel potential energy landscape for which the global minimum and the lowest lying local minimum are similar in energy, dissimilar in structure, and separated by a relatively large energy barrier. In particular, the global minimum and lowest local minimum correspond to fcc truncated octahedral and incomplete icosahedral structures, respectively.

We begin by specifying the computational ensemble. Previous work ${ }^{18}$ has shown that the heat capacity of the LJ-38 system has a primary peak in the reduced temperature range of $\mathrm{k}_{\mathrm{B}} \mathrm{T} / \varepsilon=0.165$, a value that for argon corresponds to a physical temperature of $\sim 19.8 \mathrm{~K}$. As in the $\mathrm{Ar}_{13}$ simulations discussed earlier in this section, we choose the overall temperature range for our $\mathrm{Ar}_{38}$ simulations to bracket the temperature of the major heat capacity maximum. Here, we choose to include temperatures that cover the range $10 \mathrm{~K} \leq \mathrm{T} \leq 30 \mathrm{~K}$, a region previous structural studies have shown marks the transition from solid-like to liquid-like behavior in the $\mathrm{Ar}_{38}$ cluster. The choice of the number of temperatures to include in the ensemble is somewhat arbitrary. In general, we want the number to be large enough to facilitate information flow within the ensemble, but small enough to make the simulation computationally manageable. Based on considerations described in Appendix C, we utilize a 66-temperature ensemble. The required PINS sampling is performed using the dual-chain sampling methods described in Refs. 21 and 22. Assuming that the temperatures involved are ordered, $\mathrm{T}_{1}<\mathrm{T}_{2}<\ldots,,<\mathrm{T}_{66}$, one chain consists of 11 symmetrized blocks of six temperatures each $\left(\mathrm{T}_{1-6}, \mathrm{~T}_{7-12}, \ldots, \mathrm{T}_{61-66}\right)$, while the other consists of 10 symmetrized blocks of six temperatures each that form the chain's interior and two symmetrized blocks of three temperatures that form the low and high-temperature caps for the chain, $\left(\mathrm{T}_{1-3}, \mathrm{~T}_{4-9}, \ldots, \mathrm{T}_{58-63}, \mathrm{~T}_{64-66}\right)$. The specific values of $\mathrm{T}_{1}-\mathrm{T}_{66}$ are listed in Appendix $\mathrm{C}$.

Figures 12 and 13 contain a variety of results for the $\mathrm{Ar}_{38}$ cluster obtained from two PINS simulations based on the 66-temperature ensemble described in Appendix C. Each of the two simulations contains a total of $2.0 \times 10^{6}$ moves of which the last $1.6 \times 10^{6}$ are utilized for data collection. To check that the computed results are independent of the starting conditions, one simulation is initiated using configurations corresponding to the cluster global minimum geometry, while the other is initiated using configurations corresponding to the lowest-lying icosahedral local minimum. Figures 12

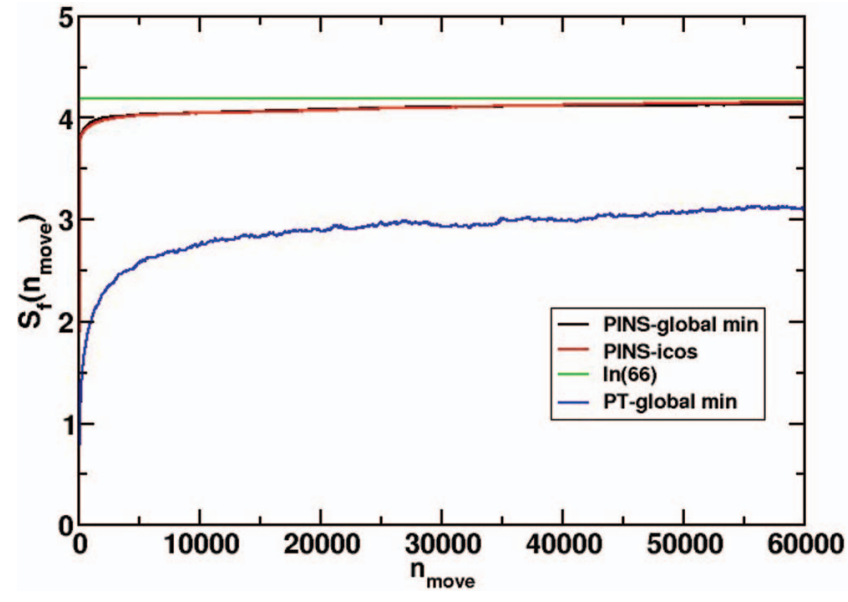

FIG. 12. Plots of the occupation entropy, $\mathrm{S}_{\mathrm{f}}$ (nmove), for 66-temperature PINS and PT simulations of the $\mathrm{Ar}_{38}$ system. The two PINS results correspond to simulations that are initiated in the global minimum geometry (black curve) or lowest-lying icosahedral minimum (red curve). The limiting $S_{\mathrm{f}}$ value of $\ln (66)$ is shown for reference.

and 13 show the occupation entropies (c.f., Eq. (2.3)) obtained from the two $\mathrm{Ar}_{38}$ simulations. Both PINS simulations converge to their uniform limits in a similar fashion. For comparison, analogous parallel tempering results are also presented in Fig. 12. The PT simulation uses the same 66-temperature ensemble, the same underlying smart Monte Carlo displacement strategy, and the same data collection procedures that are used in the PINS studies. A significant difference in the levels of performance for the PINS and PT approaches is apparent.

In Fig. 14 we show plots of the temperature dependence of the average structural order parameter, $\left\langle\mathrm{Q}_{4}(\mathrm{~T})\right\rangle$, obtained from the two PINS simulations associated with Figs. 12 and $13 . \mathrm{Q}_{4}$ is a convenient choice for distinguishing structures associated with the two major potential energy funnels of the LJ-38 system, its value ranging from near zero for icosahedral and melt structures to $\sim 0.19$ for fcc-like forms. Used by a variety of investigators, ${ }^{18,29,30}$ these order parameters are

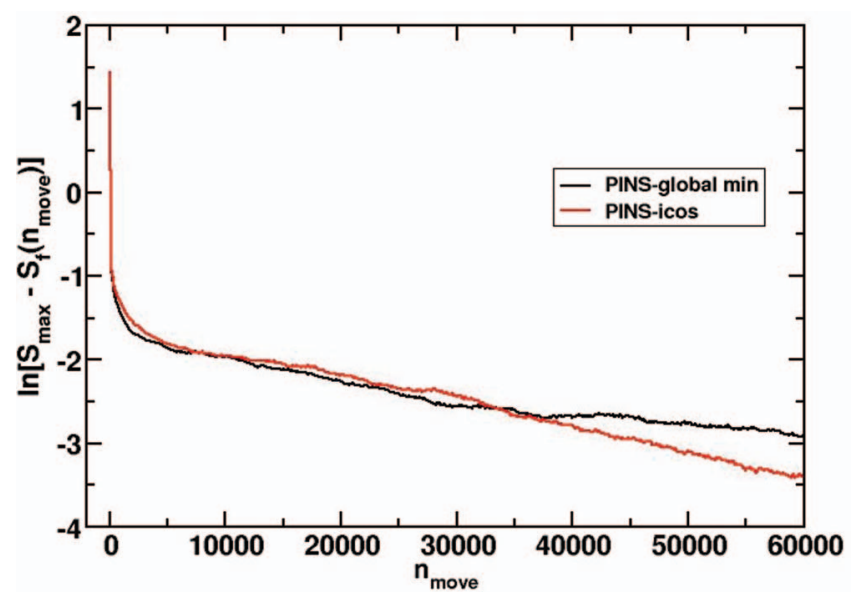

FIG. 13. Plot of $\ln \left(S_{\max }-S_{f}\right)$ for results in Fig. 12. 


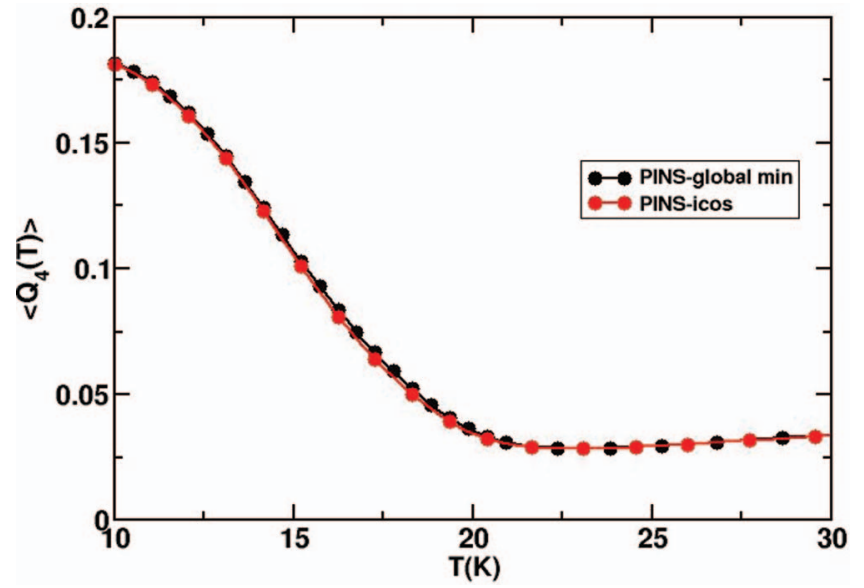

FIG. 14. A plot of $\left\langle\mathrm{Q}_{4}(\mathrm{~T})\right\rangle$ for the $\mathrm{Ar}_{38}$ cluster obtained by the PINS simulations described in the text. Results in black (red) are obtained using a simulation initialized using the fcc global minimum (icosahedral local minimum) structure. For clarity and as an aid in comparing the two simulations $\left\langle\mathrm{Q}_{4}(\mathrm{~T})\right\rangle$ only values for every other (every fourth) temperature are shown for the fcc (icosahedral) results.

defined more generally as

$$
Q_{\ell}=\left(\frac{4 \pi}{2 \ell+1} \sum_{\mathrm{m}=-\ell}^{\ell}\left|\bar{Q}_{\ell \mathrm{m}}\right|^{2}\right)^{1 / 2},
$$

where

$$
\bar{Q}_{\ell m}=\frac{1}{N_{b}} \sum_{\mathrm{r}_{i j}<\mathrm{r}_{0}} Y_{\ell \mathrm{m}}\left(\vartheta_{i j}, \phi_{i j}\right)
$$

and where the summation in Eq. (3.2) is over all pairs of atoms (bonds) for which the separation distance, $r_{i j}$, is less than a preset threshold, $\mathrm{r}_{0}$. In line with previous studies, ${ }^{18,29}$ the $\mathrm{r}_{0}$ value used in the current work is taken to be 1.391 times the Lennard-Jones $\sigma$-parameter. Again, we see equivalence in Fig. 14 for the results produced by the fcc and icosahedrally initiated simulations.

We close by returning to the issue raised at the end of Sec. II, the breakdown in the equal occupancy behavior seen in Fig. 7. Figure 15 shows the instantaneous $\mathrm{Q}_{4}$ values for the configurations visited during a somewhat longer segment of the occupation trace presented in Fig. 6. During the portion of the trace shown in Fig. $6\left(\mathrm{n}_{\text {move }}<10^{5}\right)$, we see $\mathrm{Q}_{4}$ values representative of those for the icosahedral and melt forms of the cluster, but not for those of the fcc global minimum energy structure. Because the global minimum exists preferentially at low-temperatures, its absence from the shorter occupation trace shown in Fig. 6 is the reason for the breakdown of the equal occupancy behavior seen in Fig. 7. It is interesting to note that in this example the equal occupancy measure is thus detecting the absence of visits to the global minimum. For completeness, we note that the final occupation entropies for the entire $1.6 \times 10^{6}$ move global minimum and icosahedrally initiated PINS studies associated with Figs. 12-14 are 4.1875 and 4.1895 , respectively. In the language of Sec. II, such occupation entropy values correspond to active ensemble fractions of 0.9978 and 0.9998 , values that indicate equal occupancy is indeed achieved in the two simulations.

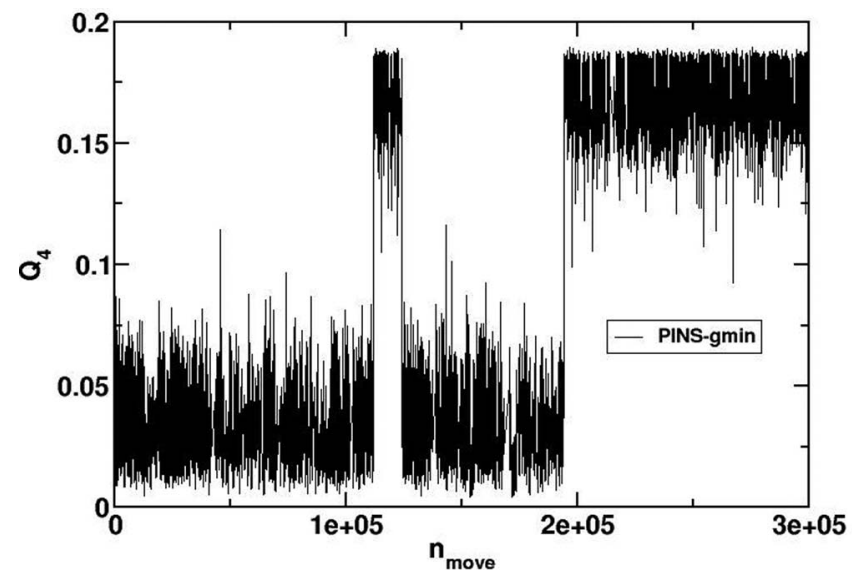

FIG. 15. $\mathrm{Q}_{4}$ values for an extended portion of the global minimum initiated occupation trace of Fig. 6.

It is useful to examine the issue of equilibration raised by Fig. 15 from a somewhat different perspective. At any given instant in the PINS simulations one can evaluate the $\mathrm{Q}_{4}$ values for the configurations in the various data streams of the computational ensemble. Such a set of values provides a rough sense of population in the various structural basins. Shown in Fig. 16 is the number of systems (out of the total of 66) for which the associated $\mathrm{Q}_{4}$ values lie below a specified threshold (here taken to be 0.09) for the $\mathrm{Ar}_{38}$ PINS simulations initiated in the global minimum and icosahedral basins. This number, $\mathrm{K}\left(\mathrm{n}_{\text {move }}\right)$, is basically a count of the number of icosahedral and melt forms. It builds from an initial value of zero as the global minimum initiated simulation proceeds, while the analogous, icosahedrally initiated result declines from an initial value of 66. After roughly 400000 moves the values of $K\left(n_{\text {move }}\right)$ for the two simulations stabilize at an average value of $\sim 45$.

Shown in Fig. 17 are block averages of $\mathrm{Q}_{4}$ values for the two PINS simulations at two specific temperatures, $T_{1}=10 \mathrm{~K}$ and $\mathrm{T}_{20}=14.9350 \mathrm{~K}$. $\mathrm{T}_{1}$ is the lowest ensemble temperature while (c.f., Fig. 15) $\mathrm{T}_{20}$ is the temperature for which $\left\langle\mathrm{Q}_{4}\right\rangle$ is roughly 0.1 , the approximate mean of $\mathrm{Q}_{4}$ 's limiting values. As with the results in Fig. 17, we see that after an initial equilibration period the block averages for the two PINS

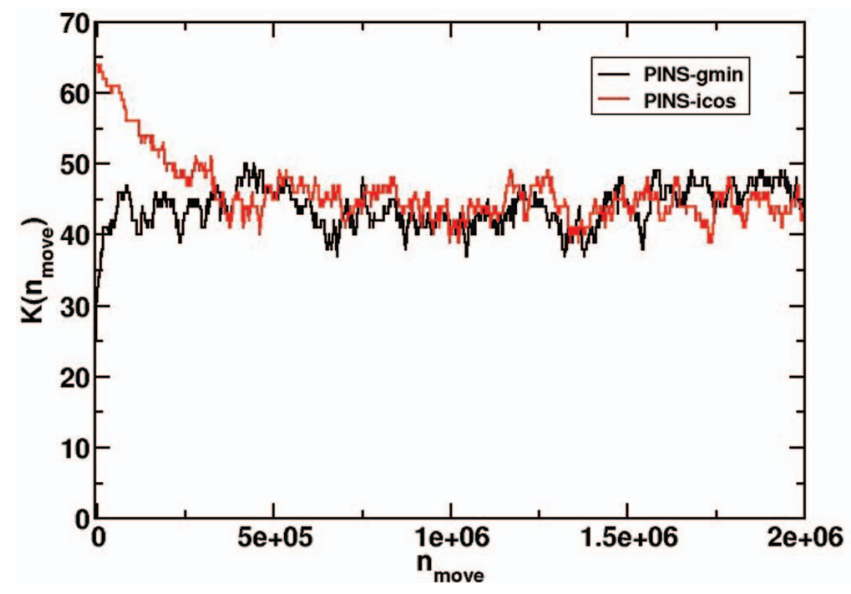

FIG. 16. A history of the number of configurations (out of 66) in the two PINS $\mathrm{Ar}_{38}$ simulations described in the text for which $\mathrm{Q}_{4} \leq 0.09$. 


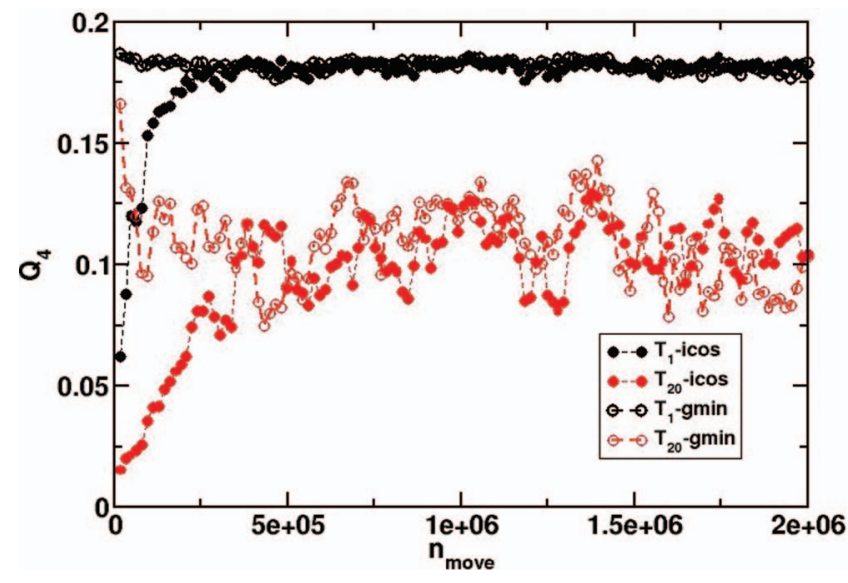

FIG. 17. Block averages of $\mathrm{Q}_{4}$ for $\mathrm{Ar}_{38}$ for $\mathrm{T}_{1}=10 \mathrm{~K}, \mathrm{~T}_{2}=14.9350 \mathrm{~K}$ for the PINS simulations described in the text.

simulations approach common limiting values. Interestingly, we see in Fig. 17 that the warm up period required to achieve stable estimates of the $\left\langle\mathrm{Q}_{4}\right\rangle$ values matches that seen in Fig. 16. In other words, the warm up period necessary to produce stable estimates of thermally averaged properties is dictated by the period required to establish the relative populations of the major energy basins. Parenthetically, we note that the equilibration period seen in Fig. 17 is the basis for our choice of 400000 move warm up periods in the PINS $\mathrm{Ar}_{38}$ simulations of this section. The values of $\left\langle\mathrm{Q}_{4}\right\rangle$ for each of the temperatures shown in Fig. 14 are constructed from the block averages of the type shown in Fig. 17 using the last $1.6 \times 10^{6}$ move portions of the two PINS simulations.

Results of the type shown in Fig. 15 represent simulation histories obtained by following a specified occupation trace throughout the simulation. Figure 18 represents another type of simulation history, one that follows a property associated with configurations that correspond to a particular temperature. Shown in Fig. 18 are the $\mathrm{Q}_{4}$ values of $\mathrm{Ar}_{38}$ configurations for a fixed temperature of $22.727 \mathrm{~K}\left(\mathrm{k}_{\mathrm{B}} \mathrm{T} / \varepsilon=0.190\right)$. The results in Fig. 18 are taken from a 10000 move segment of the larger, icosahedral minimum initiated PINS simulation after the 400000 move warm up period has been completed.

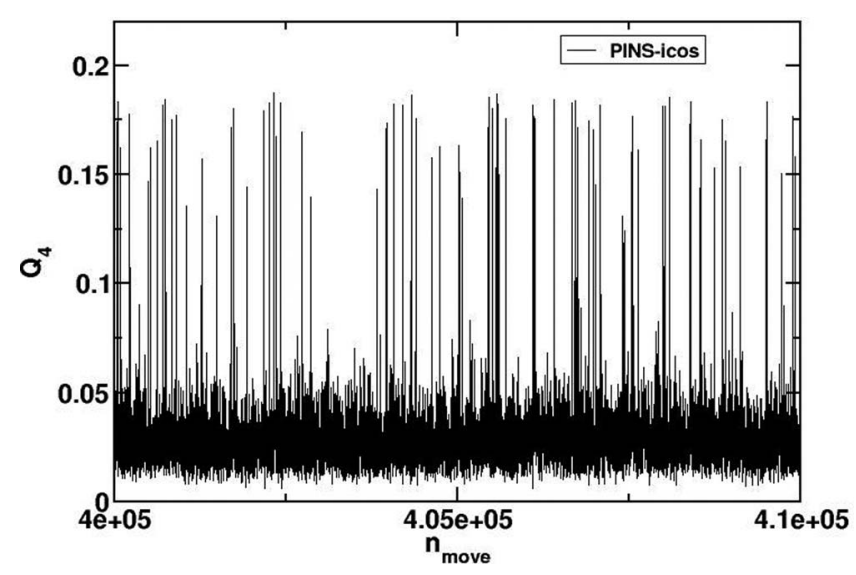

FIG. 18. Shown are the $\mathrm{Q}_{4}$ values for $\mathrm{T}=22.727 \mathrm{~K}$ for a short, post warm up portion of the icosahedral minimum initiated $\mathrm{Ar}_{38}$ PINS simulation. Compare with Fig. 11 of Ref. 29.
Analogous results have been obtained for PINS simulations initiated using the global minimum configuration. These are statistically indistinguishable from those of Fig. 18 and are not shown. The particular temperature of $22.727 \mathrm{~K}$ is chosen to permit a comparison of the results of Fig. 18 with those of conventional Monte Carlo studies (c.f., Fig. 11 of Ref. 29). Rapid switches of configurations with differing $\mathrm{Q}_{4}$ values at a fixed temperature in the PINS simulation are evident.

\section{DISCUSSION AND SUMMARY}

The ubiquitous and difficult nature of rare-event sampling issues makes the development of methods for their detection and treatment an important topic. In the present paper we have introduced a variety of occupation-based performance measures for tempering applications and have explored their utility with a number of numerical examples.

The equal occupancy result of Sec. II is the core of the present developments. Briefly summarized, equal occupancy for a tempering simulation implies that the asymptotic fraction of the total number of moves a particular configuration spends in a given data stream in a simulation that contains $\mathrm{N}_{t}$ temperatures is a constant, $1 / \mathrm{N}_{\mathrm{t}}$, independent of the choice of ensemble temperatures. Starting with the empirical observation of such behavior in parallel tempering simulations, we have demonstrated that the result is a general property of both parallel tempering as well as of PINS and infinite swapping approaches. Given that equal occupancy is a rigorous feature of such methods, its absence serves as a diagnostic for flawed and/or inadequate sampling. More generally, the rate at which equal occupancy is achieved in such a simulation offers a convenient, property-independent basis for probing both the characteristics of the sampling methods involved and the associated simulation details.

Based on these ideas we have constructed a number of occupation-based performance measures and have explored their utility using models of simple, single-component atomic clusters. We have found the resulting methods both easy to implement and effective. Based on our findings we have concluded that the occupation-based techniques presented here represent useful additions to the list of existing performance measures that have been designed to aid in the ongoing development of rare-event sampling methods.

\section{ACKNOWLEDGMENTS}

The authors gratefully acknowledge grant support of this research through the (U.S.) Department of Energy (DOE) Multiscale and Optimization for Complex Systems Program No. DE-SC0002413. N.P. wishes to thank the Swiss National Science Foundation for postdoctoral support and J.D.D. wishes to acknowledge support through the DOE departmental Program No. DE-00015561. P.D. wishes to acknowledge support from the Army Research Office (W911NF-12-10222) and P.D. gratefully acknowledges support from the National Science Foundation (DMS-1008331). The authors also wish to thank Dr. James Gubernatis, Los Alamos National Laboratory, for helpful discussions concerning the present work. 


\section{APPENDIX A: NUMERICAL METHODS}

In this appendix we describe the generic numerical methods used in the present studies including the underlying force laws and numerical methods.

The atomic-level force law in the present work is assumed to consist of pair-wise Lennard-Jones interactions between the atoms plus a center of mass confining potential to prevent cluster evaporation. Specifically, the total potential energy, $\mathrm{U}(\mathbf{r})$, for the $\mathrm{N}$-particle cluster is given by

$$
\mathrm{U}(\mathbf{r})=\mathrm{U}_{\mathrm{LJ}}(\mathbf{r})+\mathrm{U}_{\mathrm{c}}(\mathbf{r}),
$$

where

$$
\mathrm{U}_{\mathrm{LJ}}(\mathbf{r})=4 \varepsilon \sum_{\mathrm{i}<\mathrm{j}}^{\mathrm{N}}\left(\left(\frac{\sigma}{\mathrm{r}_{\mathrm{ij}}}\right)^{12}-\left(\frac{\sigma}{\mathrm{r}_{\mathrm{ij}}}\right)^{6}\right),
$$

and where

$$
\mathrm{U}_{\mathrm{c}}(\mathbf{r})=\varepsilon \sum_{\mathrm{i}=1}^{\mathrm{N}}\left(\frac{\left|\mathbf{r}_{\mathrm{i}}-\mathbf{r}_{\mathrm{cm}}\right|}{\mathrm{R}_{\mathrm{c}}}\right)^{20} .
$$

The Lennard-Jones length and energy parameters, $\sigma$ and $\varepsilon$, are taken to be $\varepsilon=119.8 \mathrm{~K}$ and $\sigma=3.405 \AA$ for argon. In the confining potential definition, $\mathbf{r}_{\mathrm{cm}}$ is the center of mass of the cluster and $R_{c}$ is an empirical constant. The $R_{c}$ values used in the present work are $2.5 \sigma$ and $2.65 \sigma$ for the $\mathrm{Ar}_{13}$ and $\mathrm{Ar}_{38}$ investigations, respectively.

All PINS simulations in the present work are generated using the dual-chain sampling techniques described in detail in Appendix B of Ref. 21. Unless otherwise stated, the chains in the simulation are constructed in the following manner. One of the chains involved is composed entirely of blocks of six adjacent temperatures, $T_{1-6}, T_{7-12}$, etc. In the second chain, the first and last blocks are composed of three-temperatures, while the remaining blocks have six temperatures each. The basic idea is to utilize symmetrized blocks that are large enough to promote appreciable mixing of temperature information, small enough to be computationally manageable, and overlap sufficiently to promote chain-to-chain information transfer.

Smart Monte Carlo (SMC) techniques ${ }^{31}$ are utilized to perform the necessary, single-temperature sampling moves in this approach. For the present argon studies each such SMC move consists of a 100 step molecular dynamics segment (each step of $10^{3}$ a.u. duration) in which the initial momenta are selected at random from equilibrium Boltzmann distributions of the appropriate temperatures. To provide a common basis for comparison, the parallel tempering simulations reported here also utilize the same SMC methods. Unless otherwise stated, all parallel tempering results are based on simulations in which at each step there is a $16 \%$ probability of an attempted swap of configurations between a randomly selected adjacent pair of temperatures.

The performance measures discussed in Sec. II are generated by suitably processing the occupation traces involved. Such occupation traces, defined in Sec. II, are a chronicle of the temperature index for the system in question at the mth step in the simulation, $\mathrm{N}(\mathrm{m})$. Once obtained, they are processed to produce the associated performance mea- sures. Computing the fluctuation autocorrelation function for a specified occupation trace (c.f., Eq. (2.4)) is entirely analogous to the task of constructing time correlation functions from molecular dynamics simulation data and thus needs no further discussion. Computing the occupation entropy (c.f., Eq. (2.3)) requires knowledge of the fractional occupancies of the various tempering data streams. To compute the occupation entropy, $\mathrm{S}_{\mathrm{f}}\left(\mathrm{n}_{\text {move }}\right)$, associated with a trace that begins in a particular temperature stream, $\mathrm{n}_{0}$, after a specified number of moves, $\mathrm{n}_{\text {move }}$, for example, we need to know the relevant fractional occupancies of the various levels, $\left\{\mathrm{f}_{\mathrm{n}}\left(\mathrm{n}_{\text {move }}\right)\right\}$, $\mathrm{n}=1, \mathrm{~N}_{\mathrm{t}}$ produced by that trace. Instead of focusing on a particular starting point, $\mathrm{n}_{0}$, it is generally more convenient to form the average of such individual occupation entropies over traces that originate in all possible starting locations. Unless otherwise noted, all occupation entropies in the present work are of this averaged type. It should also be noted that such averaged values can be computed from a single, long occupation trace by exploiting the stationary nature of the occupation traces involved.

\section{APPENDIX B: EQUAL OCCUPANCY DEMONSTRATION}

In this appendix we give two arguments to demonstrate the asymptotic equal occupancy of the temperature for PT, INS, and PINS. The first argument formalizes the one used in Sec. II. Consider a stationary, ergodic Markov process $(\mathrm{S}(\mathrm{m})$, $\mathrm{Y}(\mathrm{m})$ ) such that the first component takes values in the set $\mathrm{P}\left\{\mathrm{N}_{\mathrm{t}}\right\}$ of all permutations of $\left\{T_{1}, \ldots, T_{N_{t}}\right\}$. PT falls into this framework when the pair of temperatures for which a swap will be attempted is selected according to a randomized rule that does not depend on the past of the simulation and which allows all permutations to be realized. INS also falls into this framework where the $S(m)$ component arises through an explicit temperature-coordinate association that can be made at each time step. If the selection of partitions in a PINS scheme is done on a randomized basis, then an explicit temperaturecoordinate association must be done when switching between different partitions, and this again gives a representation for the scheme in the form described above. The asymptotic equal occupancy is not limited to methods that use temperature to index the ensemble, and can even be extended to multidimensional parameters.

With these definitions one can interpret $\mathrm{S}(\mathrm{m})$ (in the notation of Sec. II) as $\left(T_{1}(m), \ldots, T_{N_{t}}(m)\right)$. Since $S(m)$ is a permutation, for each $\mathrm{m}=1,2, \ldots$ and each $\mathrm{T}_{\mathrm{n}} \in\left\{\mathrm{T}_{1}, \ldots, \mathrm{T}_{\mathrm{Nt}}\right\}$, there is one and only one $\alpha$ such that $\mathrm{T}_{\alpha}(\mathrm{m})=\mathrm{T}_{\mathrm{n}}$. With $\mathrm{f}_{\mathrm{n}}^{\alpha}(\mathrm{M})$ defined as in Sec. II, the ergodic theorem then implies the almost sure limit

$$
\mathrm{f}_{\mathrm{n}}^{\alpha}(\mathrm{M}) \rightarrow \mathrm{f}_{\mathrm{n}}
$$

as $\mathrm{M} \rightarrow \infty$, where $\mathrm{f}_{\mathrm{n}}$ is independent of $\alpha, \mathrm{M}$, and $\mathrm{Y}(0)$. However, the fact that each $\mathrm{n}$ is identified with one and only one $\alpha$ implies that for each $\mathrm{n}$ and all $\mathrm{M}$

$$
\sum_{T_{n} \in\left\{T_{1}, \ldots, T_{N_{t}}\right\}} f_{n}^{\alpha}(M)=1
$$

from which $\mathrm{f}_{\mathrm{n}}=1 / \mathrm{N}_{\mathrm{t}}$ follows. 
While this argument applies to stationary and ergodic implementations of PT and PINS, in practice, they are often implemented in a nonstationary way. For example, in PT one may cycle repeatedly through the sequence of adjacent temperature pairs, while in the PINS scheme one could simply alternate between two different partitions (the "dual-chain" method used in Refs. 21 and 22). While each such algorithmic step corresponds to a Markov transition kernel on $(\mathrm{S}, \mathrm{Y})$, the kernels differ (e.g., depending on the particular pair of temperatures in PT or the partition of temperatures used in PINS). Thus, the processes are not stationary. Nonetheless, one can still argue that the quantity $\mathrm{f}_{\mathrm{n}}^{\alpha}(\mathrm{M})$ converges to $1 / \mathrm{N}_{\mathrm{t}}$ by an argument that uses more of the structure of the PINS, PT, and INS schemes. Here we give just a sketch of the argument.

When put in the form of transition kernels on $(\mathrm{S}, \mathrm{Y})$, there is a stationary distribution $\mu$, which satisfies detailed balance with respect to each of the different transition kernels associated with any of the PINS, PT, and INS schemes, and also this is the only such stationary distribution under all the kernels associated with a given scheme. (It is in fact the symmetrized version of the joint stationary distribution on the $\mathrm{N}_{t}$ particles, symmetrized over all temperature coordinate associations (c.f., Eq. (3.6) of Ref. 21).) It follows from the explicit form of $\mu$ that the marginal distribution on $\mathrm{S}$ is uniform on $\mathrm{P}\left\{\mathrm{N}_{\mathrm{t}}\right\}$. Using relative entropy with respect to $\mu$ as a Lyapunov function, ${ }^{32}$ one can show that, so long as each kernel associated with a given scheme is used infinitely often, the distribution $\mu(\mathrm{m})$ of the process after $\mathrm{m}$ algorithmic steps converges to $\mu$ as $\mathrm{m} \rightarrow \infty$. Denote

$$
\mathrm{g}_{\mathrm{s}}(\mathrm{M})=\frac{1}{\mathrm{M}} \sum_{\mathrm{m}=1}^{\mathrm{M}} \mathbf{1}_{\mathrm{S}(\mathrm{m}), \mathrm{s}}
$$

where

$$
\mathbf{1}_{\mathrm{S}(\mathrm{m}) \mathrm{s}}=\left\{\begin{array}{ll}
1, & \mathrm{~S}(\mathrm{~m})=\mathrm{s} \\
0, & \text { otherwise }
\end{array} .\right.
$$

Using a standard martingale argument one can show that $\mathrm{g}_{\mathrm{s}}(\mathrm{M})$ and the average (over $\mathrm{m}=1, \ldots, \mathrm{M}$ ) of the measure placed on $\mathrm{s}$ by the marginal distribution of $\mu(\mathrm{m})$ on $\mathrm{S}$ are asymptotically the same, and therefore $\mathrm{g}_{\mathrm{s}}^{\beta}(\mathrm{M}) \rightarrow 1 /\left|\mathrm{P}\left\{\mathrm{N}_{\mathrm{t}}\right\}\right|$ as $\mathrm{M} \rightarrow \infty$. The statement given in Sec. II for the equal occupancy of temperatures can then be obtained by summing over all permutations where a given coordinate is fixed.

\section{APPENDIX C: SELECTION OF TEMPERATURE ENSEMBLE}

The present discussion summarizes the design and construction of the PINS computational ensemble for studies of Sec. III. In general, the temperature range of the computational ensemble is dictated by the system and phenomenology under investigation. The lowest computational temperature is taken to be the lowest of physical interest, while the highest temperature is typically chosen to be large enough to assure a proper sampling. As a practical matter, the choice of the highest temperature amounts to selecting a value such that the simulation model in question exhibits a liquid-like behav- ior or one for which even conventional Metropolis methods prove adequate. Beyond the selection of the overall temperature range, the general design of the tempering ensemble is trade-off between the desire to minimize computational effort (smaller number of temperatures) and the desire to facilitate information flow (larger number of temperatures). The low and high-temperature limits for the $\mathrm{Ar}_{38}$ ensemble of Sec. III, $10 \mathrm{~K}$ and $30 \mathrm{~K}$, respectively, are chosen to bracket the temperature of the major heat capacity peak for the $\mathrm{Ar}_{38}$ system, ${ }^{18} \mathrm{a}$ feature that marks the boundary between solid-like and liquidlike behavior for the cluster.

In principle, once the total number, low, and high values are chosen, one can utilize the asymptotic decay rate of the occupation entropy to its known limit to optimize the selection of the remaining "interior" temperatures in the computational ensemble. In practice, we have found for the applications considered to date that the performance of the PINS approach is sufficiently robust that such a precise temperature selection process is unnecessary.

The approach we have adopted for the selection of the interior ensemble temperatures in the present work is based on the desire to enhance the rate of information flow induced by the PINS symmetrization. In practical terms, this translates into selecting the interior temperatures in such a way that the statistical importance of the various permutations that arise within the symmetrization blocks be as widely distributed as possible. In the notation of Ref. 21, if one of the blocks in the dual-chain PINS approach contains $\mathrm{N}$ total temperatures, there are $\mathrm{N}$ ! possible permutations of coordinate and temperature sets involved. As part of the PINS sampling process, the statistical weights of these permutations (c.f., Eq. (3.5) of Ref. 21) relative to the total, $\left\{\rho_{\mathrm{n}}\right\}, \mathrm{n}=1, \mathrm{~N}$ ! are computed.

TABLE II. The temperatures used in the PINS $\mathrm{Ar}_{38}$ computational

\begin{tabular}{|c|c|c|c|c|c|}
\hline $\mathrm{n}$ & $\mathrm{T}_{\mathrm{n}}$ & $\mathrm{n}$ & $\mathrm{T}_{\mathrm{n}}$ & $\mathrm{n}$ & $\mathrm{T}_{\mathrm{n}}$ \\
\hline 1 & 10.0000 & 23 & 15.7142 & 45 & 21.6364 \\
\hline 2 & 10.2597 & 24 & 15.9740 & 46 & 22.0000 \\
\hline 3 & 10.5195 & 25 & 16.2337 & 47 & 22.3637 \\
\hline 4 & 10.7792 & 26 & 16.4935 & 48 & 22.7273 \\
\hline 5 & 11.0390 & 27 & 16.7532 & 49 & 23.0909 \\
\hline 6 & 11.2987 & 28 & 17.0130 & 50 & 23.4546 \\
\hline 7 & 11.5585 & 29 & 17.2727 & 51 & 23.8182 \\
\hline 8 & 11.8182 & 30 & 17.5324 & 52 & 24.1819 \\
\hline 9 & 12.0779 & 31 & 17.7922 & 53 & 24.5455 \\
\hline 10 & 12.3377 & 32 & 18.0519 & 54 & 24.9091 \\
\hline 11 & 12.5974 & 33 & 18.3117 & 55 & 25.2727 \\
\hline 12 & 12.8572 & 34 & 18.5714 & 56 & 25.6364 \\
\hline 13 & 13.1169 & 35 & 18.8312 & 57 & 26.0000 \\
\hline 14 & 13.3767 & 36 & 19.0909 & 58 & 26.3636 \\
\hline 15 & 13.6364 & 37 & 19.3506 & 59 & 26.8182 \\
\hline 16 & 13.8961 & 38 & 19.6104 & 60 & 27.2727 \\
\hline 17 & 14.1559 & 39 & 19.8701 & 61 & 27.7272 \\
\hline 18 & 14.4156 & 40 & 20.1299 & 62 & 28.1818 \\
\hline 19 & 14.6753 & 41 & 20.3896 & 63 & 28.6364 \\
\hline 20 & 14.9350 & 42 & 20.6494 & 64 & 29.0909 \\
\hline 21 & 15.1948 & 43 & 20.9091 & 65 & 29.5454 \\
\hline 22 & 15.4545 & 44 & 21.2727 & 66 & 30.0000 \\
\hline
\end{tabular}
ensemble. 
TABLE III. Average of $\mathrm{S}_{\rho}$ values, $\left\langle\mathrm{S}_{\rho}\right\rangle$, for $\mathrm{Ar}_{38}$ PINS simulation obtained using the computational ensemble shown in Table II. For reference, the maximum values for $\mathrm{S}_{\rho}$ correspond to $\ln (3 !)=1.792$ and $\ln (6 !)=6.579$.

\begin{tabular}{lcccc}
\hline \hline & Chain-1 & & \multicolumn{2}{c}{ Chain-2 } \\
\cline { 5 - 6 } T-range & $\left\langle\mathrm{S}_{\rho}\right\rangle$ & & T-range & $\left\langle\mathrm{S}_{\rho}\right\rangle$ \\
\hline $1-3$ & 1.751 & $1-6$ & 6.256 \\
$4-9$ & 6.284 & $7-12$ & 6.307 \\
$10-15$ & 6.327 & $13-18$ & 6.345 \\
$16-21$ & 6.362 & $19-24$ & 6.375 \\
$22-27$ & 6.384 & $25-30$ & 6.383 \\
$28-33$ & 6.371 & $31-36$ & 6.352 \\
$34-39$ & 6.341 & $37-42$ & 6.351 \\
$40-45$ & 6.324 & $43-48$ & 6.285 \\
$46-51$ & 6.348 & $49-54$ & 6.385 \\
$52-57$ & 6.407 & $55-60$ & 6.393 \\
$58-63$ & 6.356 & $61-66$ & 6.371 \\
$64-66$ & 1.768 & & \\
\hline \hline
\end{tabular}

For a given set of coordinates the entropies associated with these weights, defined for each of the symmetrized blocks as

$$
\mathrm{S}_{\rho}=-\sum_{\mathrm{n}=1}^{\mathrm{N}_{\mathrm{t}}} \rho_{\mathrm{n}} \ln \left[\rho_{\mathrm{n}}\right]
$$

thus provides a measure of the dispersal of the statistical weight across the set of permutations. For example, a value of zero for $\mathrm{S}_{\rho}$ corresponds to all of the statistical weight being concentrated in a single permutation, whereas the maximum value of $\ln (\mathrm{N}$ !) signifies that each permutation carries the same uniform statistical weight.

In the case of the 66-temperature $\mathrm{Ar}_{38}$ ensemble of Sec. III we have chosen the number and distribution of temperatures in the computational ensemble so that the $S_{\rho}$ values for the various temperature blocks that are produced represent an appreciable and uniform fraction of the maximum values possible. The explicit temperatures for this ensemble are listed in Table II. Table III shows the average of the $\mathrm{S}_{\rho}$ values produced by this ensemble during the two million move simulation discussed in Sec. III that was initiated from the global minimum configuration. Although not shown, the corresponding results for the simulation initiated using the lowest-lying icosahedral local minimum configuration are statistically equivalent those of Table III.

${ }^{1}$ J. P. Valleau and S. G. Whittington, in Modern Theoretical Chemistry, edited by B. J. Berne (Plenum, New York, 1977).
${ }^{2}$ J. P. Valleau and G. M. Torrie, in Modern Theoretical Chemistry, edited by B. J. Berne (Plenum, New York, 1977).

${ }^{3}$ M. Kalos and P. Whitlock, Monte Carlo Methods (Wiley-Interscience, New York, 1986).

${ }^{4}$ D. Frenkel and B. Smit, Understanding Molecular Simulation: From Algorithms to Applications (Academic, New York, 2002).

${ }^{5}$ N. Metropolis, A. W. Rosenbluth, M. N. Rosenbluth, A. H. Teller, and E. Teller, J. Chem. Phys. 21, 1087 (1953).

${ }^{6}$ D. J. Earl and M. W. Deem, Phys. Chem. Chem. Phys. 7(23), 3910-3916 (2005).

${ }^{7}$ C. J. Geyer, in "Computing science and statistics," Proceedings of the $23 \mathrm{rd}$ Symposium on the Interface, edited by E. M. Keramigas (Interface Foundation, Fairfax, 1991), pp. 156-163.

${ }^{8}$ C. J. Geyer and E. A. Thompson, J. Am. Stat. Assoc. 90, 909 (1995).

${ }^{9}$ K. Hukushima and K. Nemoto, J. Phys. Soc. Jpn. 65(6), 1604 (1996).

${ }^{10}$ D. A. Kofke, J. Chem. Phys. 117(15), 6911 (2002).

${ }^{11}$ C. Predescu, M. Predescu, and C. V. Ciobanu, J. Phys. Chem. B 109(9), 4189 (2005).

${ }^{12}$ C. Predescu, M. Predescu, and C. V. Ciobanu, J. Chem. Phys. 120(9), 4119 (2004).

${ }^{13}$ D. Sabo, M. Meuwly, D. L. Freeman, and J. D. Doll, J. Chem. Phys. 128(17), 174109 (2008).

${ }^{14}$ H. G. Katzgraber, S. Trebst, D. A. Huse, and M. Troyer, J. Stat. Mech.: Theory Exp. 2006, P03018.

${ }^{15}$ D. Sindhikara, Y. L. Meng, and A. E. Roitberg, J. Chem. Phys. 128(2), 024103 (2008)

${ }^{16}$ D. J. Sindhikara, D. J. Emerson, and A. E. Roitberg, J. Chem. Theory Comput. 6, 2804 (2010).

${ }^{17}$ F. Calvo, J. Chem. Phys. 123(12), 124106 (2005).

${ }^{18}$ J. P. Neirotti, F. Calvo, D. L. Freeman, and J. D. Doll, J. Chem. Phys. 112, 10340 (2000).

${ }^{19}$ M. Athenes and F. Calvo, ChemPhysChem 9, 2332 (2008).

${ }^{20}$ I. Coluzza and D. Frenkel, ChemPhysChem 6, 1779 (2005).

${ }^{21}$ N. Plattner, J. D. Doll, P. Dupuis, H. Wang, Y. Liu, and J. E. Gubernatis, J. Chem. Phys. 135, 134111 (2011).

${ }^{22}$ P. Dupuis, Y. Liu, N. Plattner, and J. D. Doll, SIAM, Multiscale Modeling and Simulation 10, 986-1022 (2012).

${ }^{23}$ A. Kone and D. A. Kofke, J. Chem. Phys. 122, 206101 (2005).

${ }^{24}$ J. P. Neirotti, D. L. Freeman, and J. D. Doll, Phys. Rev. E 62, 7445 (2000).

${ }^{25}$ D. Thirumalai, R. D. Mountain, and T. R. Kirkpatrick, Phys. Rev. A 39, 3563 (1989).

${ }^{26}$ D. D. Frantz, D. L. Freeman, and J. D. Doll, J. Chem. Phys. 93, 2769 (1990).

${ }^{27}$ S. Trebst, M. Troyer, and U. H. E. Hansmann, J. Chem. Phys. 124, 174903 (2006).

${ }^{28}$ D. J. Wales, Energy Landscapes With Applications to Clusters, Biomolecules and Glasses (Cambridge University Press, Cambridge, 2003).

${ }^{29}$ J. P. K. Doye, M. A. Miller, and D. J. Wales, J. Chem. Phys. 111(18), 84178428 (1999).

${ }^{30}$ P. J. Steinhardt, D. R. Nelson, and M. Ronchetti, Phys. Rev. B 28, 784 (1983).

${ }^{31}$ P. J. Rossky, J. D. Doll, and H. L. Friedman, J. Chem. Phys. 69, 5628 (1978).

${ }^{32}$ F. Spitzer, "Random fields and interacting particle systems," Notes on lectures given at 1971 MAA Summer Seminar, Williamstown, MA (Mathematical Association of America, Washington, DC, 1971). 